\title{
Tensiones y desigualdades en las interacciones áulicas en estudiantes universitarios
}

\section{Tensions and inequalities in university students' classroom interactions}

\author{
Rita Virginia Ramos-Castro \\ María Minerva López-García \\ Universidad Autónoma de Chiapas, Chiapas, México
}

\section{Resumen}

Objetivo: Comprender las interacciones áulicas en el estudiantado de la Licenciatura en Bibliotecología de la Universidad Autónoma de Chiapas. Método: La metodología utilizada fue de tipo etnográfico, centrándose de manera particular en la observación del trabajo en el aula de 48 estudiantes, repartidos en seis grupos y doce profesores. Se llevaron a cabo entrevistas colectivas a los primeros, en tanto que se realizó de forma individual a los segundos. Método: La metodología utilizada fue de tipo etnográfico, centrándose de manera particular en la observación del trabajo en el aula de 48 estudiantes, repartidos en seis grupos y doce profesores. Se llevaron a cabo entrevistas colectivas a los primeros, en tanto que se realizó de forma individual a los segundos. Resultados: El profesorado en general realiza una serie de acciones como las de clasificar previamente al estudiantado en términos del tipo de interacción establecida con el conocimiento teórico metodológico de la disciplina. Muchos de estos estudiantes tienen un origen sociocultural de medio a escasos recursos con un manejo de un código lingüístico restringido, por lo que acceder al conocimiento disciplinario resulta complejo. Esta condición de desigualdad desde el punto de vista de los profesores debería resultar en la exclusión del sistema educativo de estudiantes que no cumplan con lo aparentemente ideal, porque dificulta el proceso enseñanza-aprendizaje. Discusión y Conclusiones: Las interacciones asimétricas entre el profesorado y el estudiantado acentúan las condiciones de desigualdad y provocan tensión en la práctica pedagógica cotidiana. Esto abre la discusión sobre la ausencia de formación docente con una falta de sensibilización por parte del profesorado para comprender la realidad educativa en la que se encuentran inmersos que no permiten la ayuda pedagógica necesaria para favorecer aprendizajes significativos ante la presencia de un código restringido y un capital cultural que no le permite acceder a los contenidos escolares.

Palabras clave: Desigualdad educativa y social, educación superior, códigos lingüísticos, práctica docente.

\begin{abstract}
Objective: To understand the classroom interactions of the students of the Bachelor's degree in Librarianship at the Autonomous University of Chiapas. Method: Ethnographic methodology was used, with a specific focus on the analysis of the work done in the classroom by 48 students, divided into 6 groups, and 12 teachers. Collective interviews were conducted with the former, while the latter were individually interviewed. Results: The teachers carry out a series of activities such as classifying students according to the type of interaction established with the theoretical-methodological knowledge of the field in advance. Most of these students had a middle or low socioeconomic status with a restricted linguistic code and, as a result, accessing disciplinary language was complex for them. This inequality condition from the teachers' perspective led to the exclusion of students who failed to meet the ideal from the education system because it hindered the teaching-learning process. Discussion $y$ Conclusion: Unsymmetrical interactions between teachers and students enhance the inequality conditions and cause tension in the daily pedagogic practice. This leaves room for discussion regarding the lack of teacher training and also the teachers' lack of awareness with regard to the understanding of the educational reality they are immersed in, which does not allow for the provision of the pedagogical assistance required to favor meaningful learning in the presence of a restricted code and a cultural capital that prevents them from accessing the academic curricula.
\end{abstract}

Keywords: Education and social inequality, higher education, linguistic codes, teaching practice.
Open Access:

ISSN: $0124-212$

E-ISSN: $2665-2420$

ARTÍCULO RESULTADO DE INVESTIGACIÓN
Copyright @ 2020 By Educación y Humanismo

Editor:

Patricia Martínez Barrios Universidad Simón Bolívar

Correspondencia

María Minerva López minerva@unach.mx

Recibido: 03-11-2019 Aceptado: 17-12-2019 En línea desde: 26-03-2020 


\section{Introducción}

La desigualdad dentro de las aulas en las sociedades capitalistas parece haber recibido carta de naturalización para percibirse como un hecho normal entre aquellos con posibilidades de acceso a los recursos distribuidos de forma poco equitativa con los que menos tienen, dividiéndose la sociedad en clases, manteniendo el mito del individualismo como forma de alcanzar mayores grados de competitividad.

Las relaciones asimétricas se manifiestan en un conjunto de condiciones marcadas por la pobreza extrema y el dispendio de quienes, se puede decir, lo tienen todo. La escuela, en este aspecto ha jugado un papel importante para reproducir estos esquemas de desigualdad, desde la lectura de los sociólogos. Por ejemplo, Parsons (1966) señalaba que todas las instituciones tienden a reproducir los patrones establecidos, teniendo a la escuela como el espacio de ejecución de estas normas y pautas de conductas. Desde las teorías de la reproducción en el caso particular de la de Bernstein, en donde se describen los mecanismos de estructuración simbólica de los discursos pedagógicos.

Según Lera, Martínez, Ochoa y Rivas (2017) en la teoría de Bernstein se comprende que

Cada pedagogía privilegia ciertos tipos de producción simbólica, según su adecuación a tales principios reguladores. El discurso pedagógico dominante favorece lo que Bernstein denomina "código elaborado" de comunicación, propio de las clases medias y desprestigia el "código restringido" de las clases obreras. ( $p$. 139).

Desde esta perspectiva teórica se comprende cómo en la escuela se desarrollan interacciones significativas que se producen de manera consciente $o$ inconsciente entre los actores del proceso enseñanza aprendizaje (profesor-estudiante, estudiante-estudiante), determinando a la vez las formas de pensar, sentir y actuar. Por esta razón es la escuela donde se orienta la conducta de los estudiantes, su agrupación, la jerarquía escolar, la evaluación de los docentes, las formas de acreditación, el currículum, los ritos, costumbres de la vida social en la escuela. Por ello, las habilidades que presenta cada sujeto participante en la práctica educativa, van en algún momento dado a propiciar desigualdad escolar. Incluso la vestimenta influye para definir diferencias de condiciones económicas. Aun más, si se toma en cuenta que vivimos en un mundo natural donde no existen igualdades sino únicamente semejanzas; por lo que, la desigualdad es rectora de la existencia universal (Carrillo, 1982).

Gil-Antón (2016), analiza, en el caso de México, el problema de la desigualdad educativa desde las reformas que se implementan en el sistema educativo, demostrando a través de una serie de cifras el asunto de la equidad, más como una aspiración que como una realidad. Si bien la función social de la educación es la de promover cambios desde el 
imaginario de la movilidad (Bazdrech, 2001, como se citó en Márquez, 2016) y aunque en nuestro país se le atribuya al sector educativo un papel central, proponiendo reformas para una mejor educación que en la administración gubernamental con mucha incertidumbre sobre las posibilidades reales de cambio.

La diversidad sociocultural de los estudiantes dentro del contexto escolar es un fenómeno que podría entenderse como algo positivo, sino estuviera matizada por la mirada de los otros a través de los lentes de sus creencias y prejuicios en un ejercicio cotidiano de dominación del hombre por el hombre. La riqueza que podría tener la diversidad se ve opacada por la idea de lo diferente como equivalente a extraño, negativo o anormal. La premisa sobre la que se basa la idea de la riqueza, implica el reconocimiento de la diversidad y se sustenta en el hecho de que los aprendizajes más significativos del estudiantado no ocurren solo en las aulas y los muros escolares, sino al exterior, en el día a día de convivencia en un mundo desigual y diverso. Sin embargo, esta diversidad se comprende desde las políticas educativas como retos y desafíos para hacer frente a condiciones tan disímiles para brindar educación con equidad.

Si bien se ha atendido parcialmente el asunto de la cobertura, tal como lo propone la Organización de las Naciones Unidas para la Educación, la Ciencia y la Cultura (UNESCO, 2011) de cumplir con el compromiso del derecho a la educación de todos, también se observan una serie de cuestiones sin resolver en los niveles educativos desde la educación inicial hasta el nivel superior como lo es el hecho de no garantizar la permanencia y mucho menos por el egreso con una educación que verdaderamente desarrolle las habilidades para que los futuros profesionales puedan insertarse en la sociedad, con las competencias que les permita resolver los problemas a los que se enfrenten.

La división de la sociedad en clases, ha marcado la distancia entre las personas que pertenecen a sectores menos favorecidos socioeconómicamente y con verdaderos predicamentos para acceder a la cultura escolar; tal es el caso de la educación que reciben los estudiantes procedentes de familias indígenas, campesinas, obreras y urbanas marginadas con un proceso educativo que se desarrolla en forma poco satisfactoria. A su vez, esto se traduce, a largo plazo, en una inequitativa distribución de oportunidades escolares. Esta condición aparece naturalizada en las sociedades capitalistas, además, propicia un trato desigual que se traduce también en desigualdad escolar, impidiendo con esto el óptimo desempeño académico porque en su desenvolvimiento escolar, a pesar de haberles permitido acceder a un nivel superior, estos estudiantes muestran que no han adquirido adecuadamente las estructuras relacionadas con el pensamiento formal por el manejo del lenguaje simbólico.

Por lo anterior, se sostiene que las desigualdades del estudiantado cuando llegan al nivel de educación superior serán remarcadas y sancionadas en su vida escolar y son producto de la condición de clase a la que pertenecen. De ahí la importancia de llevar a cabo una investigación con estudiantes de la Licenciatura en Bibliotecología y Gestión de 
Información de la Facultad de Humanidades de la Universidad Autónoma de Chiapas.

El ejercicio investigativo llevado a cabo, permitió identificar una serie de dimensiones y categorías para procesar los datos recabados a través de la etnografía como método de indagación para mostrar el entramado que se desarrolla a través de las interacciones áulicas de estudiantes y profesores. Para efectos de esta investigación se centró la atención particularmente en la percepción que tiene los estudiantes sobre sí mismos, sus profesores, la licenciatura y la Universidad a la que pertenecen. Estos elementos marcan significativamente el tipo de interacción comunicativa que ocurre al interior de las aulas, reflejadas en la evaluación de los docentes los cuales tienen su propia mirada del estudiantado.

Según Perret-Clermont (1979), es a través de la evaluación donde se concretan las desigualdades, pues considera que sigue siendo una utopía pedagógica ofertar una escuela para todos. Sostiene la idea de una contradicción interna que resulta central en la escuela, al tratar de ofrecer oportunidades iguales de acceso sometiendo a diferentes estudiantes bajo las mismas reglas. Ya sea por causas naturales y/o socioculturales, los estudiantes son desiguales y la escuela, con la intención de aplicar medidas de rendimientos iguales, provoca que los individuos de las clases populares deban realizar un esfuerzo mayor para entender o asimilar la cultura escolar, generándose una especie de reeducación. Por lo que, lo ideal sería educar en la desigualdad, para así, al final del proceso pedagógico, se logre, aunque sea mínima, una igualdad en cuanto al desarrollo cognitivo, puesto que, en cuestiones culturales, los estudiantes tienden a conservarlo en su mayoría al momento de egresar de los nichos escolares.

Asimismo, Lutte (1991) señala que la escuela "humilla" con frecuencia a los integrantes de las clases desfavorecidas, mencionando consecuencias destacadas como el fracaso escolar, las notas bajas y sobre todo los juicios de los profesores, que terminan convenciéndolos de su incapacidad para de poder sobresalir en sus estudios, debiendo conformarse con un trabajo de acuerdo con su estrato social sin aspiraciones a una movilidad vertical ascendente.

A este respecto, Bartollucci (2000) sostiene que la familia influye de manera directa en el ingreso al nivel superior, en particular los padres, puestos que estos le otorgan a la escuela un valor universal como la mejor opción y herencia. Desde esta perspectiva, concluir una profesión, es sinónimo de éxito en la vida o por lo menos daría la posibilidad de ser una persona productiva para su familia y la propia sociedad. Aunque durante mucho tiempo se mantuvo el mito de la movilidad social a través de la educación, es evidente que la crisis del empleo ha generado desencanto en egresados, al encontrarse con un panorama desolador al subemplearse o autoemplearse. En tanto la escuela convoca a la competencia por ser el mejor, el primer lugar, el sobresaliente, para alcanzar mejores oportunidades laborales. 
Márquez (2016), al referirse al problema de la desigualdad educativa señala:

Se encuentran estrechamente asociadas a la distribución del ingreso en México, pues en el 20 por ciento de los hogares más pobres el porcentaje de asistencia escolar se encuentra sistemáticamente por debajo del porcentaje que alcanza en el 20 por ciento de los hogares con mayores recursos. (p. 6).

Las instituciones educativas exigen el cumplimiento de patrones conductuales que no son bien recibidos por los estudiantes, pero forman parte del ritual y discurso de la cultura escolar íntimamente asociada con el éxito social y la mejora de la calidad de vida. Estos elementos de la cultura escolar son a los que no pueden acceder todos porque el medio del que proceden les negó esas pautas conductuales y de conocimiento. Elementos organizacionales tales como las expectativas sociales de los sujetos (padres, profesores, estudiantes y sociedad), el diseño del proceso de enseñanza-aprendizaje y la evaluación (Redondo, 1997) pueden permitir el análisis para entender el fracaso escolar de las clases menos favorecidas.

Bernstein, (como se citó en Feito s/f), desde el punto de vista sociolingüístico, identifica la existencia de dos tipos de códigos que subyacen en la comunicación entre profesores y estudiantes; encontrando rasgos diferenciados a partir de la clase social a la que cada uno pertenece, un código restringido y un código elaborado, los cuales son elementos de análisis para predecir las posibilidades de éxito futuro en una escuela. Desde su punto de vista, la institución escolar privilegia una cultura denominada académica con el uso común de un código elaborado independientemente de la asignatura que se trate.

Feito (s/f) haciendo referencia a la teoría de Bernstein propone el siguiente cuadro donde se enmarcan las diferencias entre el código elaborado y el código restringido.

Tabla 1: Diferencias entre código elaborado y restringido

Código elaborado

Precisión de la organización gramatical y de la sintaxis.

Matices lingüísticos vehiculados por una construcción de la frase gramaticalmente compleja, y especialmente por la utilización de una serie de conjunciones y de proposiciones subordinadas.

\section{Código restringido}

Uso frecuente de frases gramaticalmente simples, cortas, a menudo sin acabar; una construcción sintáctica pobre con una forma verbal que expresa el estado de ánimo.

Uso simple y repetitivos de conjunciones (porque, entonces).

Uso frecuente de preposiciones, las cuales indican relaciones lógicas, así como de preposiciones que indican la proximidad espacial y temporal.
Uso frecuente de preguntas y órdenes cortas.

Uso limitado y rígido de adjetivos y adverbios.

Uso frecuente de pronombres impersonales "se puede afirmar."

Elección rigurosa de los adjetivos y los adverbios.

Uso infrecuente de pronombres personales

Frases formuladas como preguntas que establecen una singularidad simpatética, v.g.; 
no, verdad

Una afirmación de hecho se utiliza tanto como razón como conclusión, o mejor dicho, la razón y la conclusión se confunden para producir una afirmación categórica. "Haz lo que se te dice, no vas a salir porque lo digo yo".

Uso frecuente de frases idiomáticas.

Fuente: Tomado de Feito (s/f).

Las condiciones materiales de vida cotidiana están vinculadas directamente con el uso de códigos restringidos y elaborados porque la convivencia con artefactos culturales diversos, propios de cada clase social, les significa de distinto modo. Las relaciones de trabajo en la interacción con los otros, proveen esas posibilidades de ampliar la cosmovisión o restringirla, según sea el caso.

Si bien, no se puede generalizar entre la clase obrera a todo trabajador rural o urbano, parece ser que comparten las mismas limitaciones de acceso para considerar igual de importantes los elementos de la cultura en la cual se insertan. Así se encuentra que se mantienen las costumbres y tradiciones, muchas de estas basadas en creencias que no estimulan los procesos cognitivos para analizar críticamente la realidad.

La teoría general de los códigos, sirve de referente a Bernstein y Díaz (1985) para explicar la teoría general del discurso, señalando:

El discurso aparece entonces como un producto de la división social del trabajo de las categorías implicadas en su producción, (sean estas agentes o discursos) y de sus correspondientes relaciones sociales. Debemos notar aquí que, las categorías sociales (agentes o discursos) están constituidas por la división social del trabajo y las prácticas que se constituyen en las relaciones sociales. (p. 7).

Desde este planteamiento la pertenencia a una clase, trae consigo un conjunto de prácticas con artefactos propios que le corresponden a su condición de vida y con sus propios códigos, existiendo otros a los que no tiene acceso, pero que son importantes por las oportunidades de pertenecer a otras categorías sociales dependiente de los códigos. Por su parte, Austin (1981) menciona:

En el transcurso de nuestras interacciones, no solo hacemos descripciones del mundo sino que nuestros enunciados ejercen funciones específicas y cumplen objetivos determinados. El lenguaje no sólo tiene como función la descripción de la realidad, sino que permite realizar acciones sociales. En la interacción, el lenguaje actúa y es necesario tomarlo en cuenta para comprenderla. (p. 15).

Ambos teóricos hacen especial énfasis en el uso del lenguaje como capital lingüístico que sirve como materia prima en el trabajo escolar, el cual es necesario activar tanto para comprender lo que se lee como para comunicar apropiadamente el saber. Este lenguaje aprendido en el hogar se hace evidente en las interacciones áulicas que pueden determinar el grado de convivencia y asociación con los compañeros y con los profesores. 
Sin embargo, Blanco (2017) utiliza el concepto movilidad educativa limitada para explicar esta situación de desigualdad, indicando que los recursos, aunque no siguen un patrón de distribución excluyente, no hacen una diferencia en los aprendizajes que se compare a los factores estructurales. Por ello señala

Coexisten patrones de movilidad y reproducción, con predominio de los primeros. Esto no quiere decir que no haya fluidez absoluta de oportunidades educativas; las desigualdades son persistentes, pero: a) existen patrones de acumulación de recursos y despliegue de prácticas que siguen una lógica más abierta en relación con el origen social que propone la TR; b) esta apertura es especialmente notoria para el acceso a recursos de tipo educativo, por contraposición a la relación con la "alta cultura"; c) no obstante, la eficacia de estos recursos para obtener ventajas educativas es limitada, en comparación con la que proveen los factores estructurales; y d) en consecuencia, las desigualdades asociadas a los factores estructurales se reproducen en el ámbito educativo a pesar de la apertura relativa en el acceso a los recursos. (p. 762).

Blanco (2017) explica esta movilidad educativa limitada en razón de una elevada desigualdad social que se combina con un acaparamiento de mecanismos corporativos de reproducción que genera posibilidades de segregar procesos de escolarización, haciendo que, en los sectores privilegiados, se exprese la necesidad de recurso a capitales y esquemas específicamente educativos. En el caso de los sectores medios, con una movilidad reciente, no se logra la acumulación significativa de capital cultural y tampoco se puede desarrollar habitus de la alta cultura, por lo que estos recursos tampoco tienen un valor de privilegio.

Así, también Martínez-Rizo (2012), hace referencia a esta condición de desigualdad donde las escuelas que atienden a sectores desprotegidos tienen menos recursos financieros para hacer frente a este problema, lo que conlleva el riesgo de incrementar esta condición que no favorece la equidad, señala que:

A diferencia de lo que debería ocurrir, las escuelas mexicanas no compensan las desventajas del contexto de los hogares más pobres, sino que más bien las refuerzan, ya que los alumnos de circunstancias más vulnerables en el hogar son atendidos en escuelas con mayores carencias. (p. 44).

Bajo estas circunstancias lo predecible para estos tipos de estudiantes que ingresan al nivel superior es la inexistencia de condiciones que le permitan acceder de manera efectiva a los requerimientos que le son exigibles desde la cultura escolar

\section{Método}

En este artículo se presentan los resultados de las observaciones de las interacciones áulicas y las entrevistas realizadas al profesorado y estudiantado de la Licenciatura en Bibliotecología y Gestión de la Información de la Universidad Autónoma de Chiapas. Desde un enfoque etnográfico (Mauss, 1974; Malinovski, 1995, Goetz y Lecompte, 1988) la 
investigación centró su interés en las pautas comunicativas de los profesores hacia los estudiantes en el salón de clases, mediadas por el contenido escolar y los recursos didácticos utilizados para favorecer el aprendizaje disciplinar. El enfoque etnográfico en lo educativo permite efectuar, desde el punto de vista de Martínez (2012), una descripción de los acontecimientos tanto a nivel individual como en el colectivo, que den lugar a la interpretación en términos de la cultura de la cual forma parte el o los fenómenos investigados. Dentro de los principales requerimientos básicos para llevar a cabo estudios de carácter etnográfico, Capocasale (2015) sugiere, tomar en consideración una serie de elementos que permitan captar los significados de la población en estudio. Considera importante en primer lugar, la observación directa, en segundo lugar una estancia relativamente larga del investigador en el escenario de investigación, en tercer lugar, un volumen considerable de investigación y en cuarto lugar una contextualización amplia del fenómeno a investigar.

Maturana y Garzón (2015) destacan la importancia de la etnografía educativa, porque el estudio de los escenarios de interacción humana, cultura escolar y cotidianidad, así como dinámicas y necesidades, requiere de métodos alternativos de investigación. Partiendo de esto se realizaron observaciones a tres grupos de la Licenciatura que se encontraban cursando el tercer, quinto y octavo semestre, en el periodo enero-junio de 2018. Asimismo, se realizaron entrevistas no estructuradas a ocho profesores que estaban a cargo de los cursos en estos grupos.

La interacción en el aula, se analizó a partir de las estructuras de participación propuesta por Rockwell (1995) porque en cada clase puede ocurrir una enorme variedad de situaciones a través de las cuales se comunican estudiantes y profesores, desde el supuesto de que el segundo dirige controla, legitima y desaprueba la respuesta verbal o no verbal de sus estudiantes, advirtiéndose, por lo tanto, una relación asimétrica. También esta autora señala que existen diversos estilos para interactuar con el grupo desde el trato formal, hasta el agresivo, tolerante o desigual.

\section{El contexto de la investigación}

La Licenciatura en Bibliotecología , ofertada en la Universidad Autónoma de Chiapas (UNACH), surge a partir de dos momentos: El primero, en las gestiones realizadas por la Sección Chiapas de la Asociación Mexicana de Bibliotecarios AC (AMBAC), en el período que va de 1988 a1990. Durante este, la mesa directiva trabajó en una propuesta para la planeación integral de las bibliotecas en el estado de Chiapas. De acuerdo con Chacón (2003), dentro de las propuestas planteadas al gobernador de la entidad se destacaron las siguientes:

- Realizar en Chiapas las Jornadas Mexicanas de Biblioteconomía y Archivonomía. 
- Establecer un convenio institucional con la Escuela Nacional de Biblioteconomía (SEP) o la UNAM (Facultad de Filosofía y Letras, Licenciatura en Bibliotecología) para establecer un proceso de formación de profesionales a nivel licenciatura o maestría en un sistema abierto

- $\quad$ Establecer la primera Escuela de Biblioteconomía del sureste con sede en Tuxtla Gutiérrez, Chiapas, con base en la infraestructura educativa, bibliotecaria y de recursos humanos existentes en el estado.

El segundo momento fue durante la celebración de las XXII Jornadas Mexicanas de Biblioteconomía, llevadas a cabo en la ciudad de Tuxtla Gutiérrez, Chiapas, los días 13,14 y 15 de mayo de 1991; en las que se rindió homenaje al maestro Roberto A. Gordillo Gordillo, por el cumplimiento de sus 47 años de su quehacer en el servicio bibliotecario. En su discurso de agradecimiento realizado durante este evento, el maestro propuso al Gobierno del Estado y a la Administración de la UNACH, la posibilidad de presentar un proyecto para instituir la licenciatura en Bibliotecología en la máxima casa de estudios chiapaneca. Afortunadamente la propuesta fue aprobada y aceptada por la universidad quedando esta acción social plasmada en el Proyecto Académico 1991-1994 del Lic. Jorge Arias Zebadúa, en ese entonces rector de la UNACH, siendo anunciada en el mes de junio de 1991 como una opción más en el futuro profesional de la comunidad estudiantil. De este modo la licenciatura quedó concretamente est ablecida en septiembre del año 1992 en la Facultad de Humanidades (UNACH, 1992).

El plan de estudios fue organizado para ser cursado en nueve semestres, distribuidos en 49 materias con un total de 429 créditos, de los cuales 328 corresponden a horas teóricas y 101 a horas prácticas. El propósito de la licenciatura es:

Formar profesionales que, mediante la comprensión de los fundamentos filosóficos, científicos y técnicos de la especialidad, así como de otras disciplinas afines y la aplicación racional de las teorías y técnicas respectivas, satisficieran las necesidades de información documental de los usuarios y sus intereses en cuanto autoeducación y recreación (UNACH, 1992, p. 36).

En este plan, se esperaba formar bibliotecarios profesionales capaces de satisfacer las necesidades e intereses de información documental, autoeducación y recreación a partir de la comprensión de los fundamentos filosóficos, científicos y técnicos de la especialidad; así como de otras disciplinas afines y la aplicación racional de las teorías y técnicas respectivas.

Con relación a los propósitos curriculares del programa se establecieron, tomando como referencia las habilidades para el análisis, en la comprensión de la problemática intelectual, institucional, social y cultural que requiere de identificarla para la adquisición y acrecentamiento del saber, al igual que para la explicación de los orígenes y fundamentos de las ciencias, las letras y la tecnología, como coadyuvante al desarrollo de la vida social y la elevación de sus niveles de bienestar. 
Otro propósito importante se relaciona con el desarrollo de actitudes y habilidades necesarias para la comprensión y aplicación de principios y técnicas de administración para que puedan usarla con fines de adquisición, preservación y difusión del pensamiento humano, así como fundamentar el conocimiento desde bases humanísticas, técnicas y desarrollar actitudes y habilidades que lo formen para asesorar y orientar a los usuarios en la utilización y aprovechamiento óptimo de los recursos y servicios bibliotecarios.

\section{Planta docente y población estudiantil}

La planta docente está conformada por dieciocho profesores, de los cuales $72.2 \%$ tienen perfil en el área bibliotecológica, mientras que $27.7 \%$ se ubica con formación en disciplinas tales como Pedagogía, Sistemas Computacionales, Contaduría Pública y Derecho. Del total de profesores, $38.8 \%$ son de tiempo completo, mientras que $61.1 \%$ son de tiempo parcial o conocidos por asignatura. Estos últimos, de acuerdo a Buendía, Acosta y Gil Antón (2019) son considerados como un grupo social no re-conocido ni en sus rasgos y trayectorias; ni teórica, normativa, ni empíricamente, son los ausentes en las políticas públicas.

La población estudiantil, en comparación con las otras cinco licenciaturas que se ofertan en la Facultad de Humanidades, Campus VI, es la que tiene menor demanda, históricamente el número mayor oscila entre cinco a nueve estudiantes en promedio por grupo. Actualmente se encuentran cursando un total de 48 estudiantes distribuidos de la siguiente manera:

Grafico 1. Estudiantes por semestre

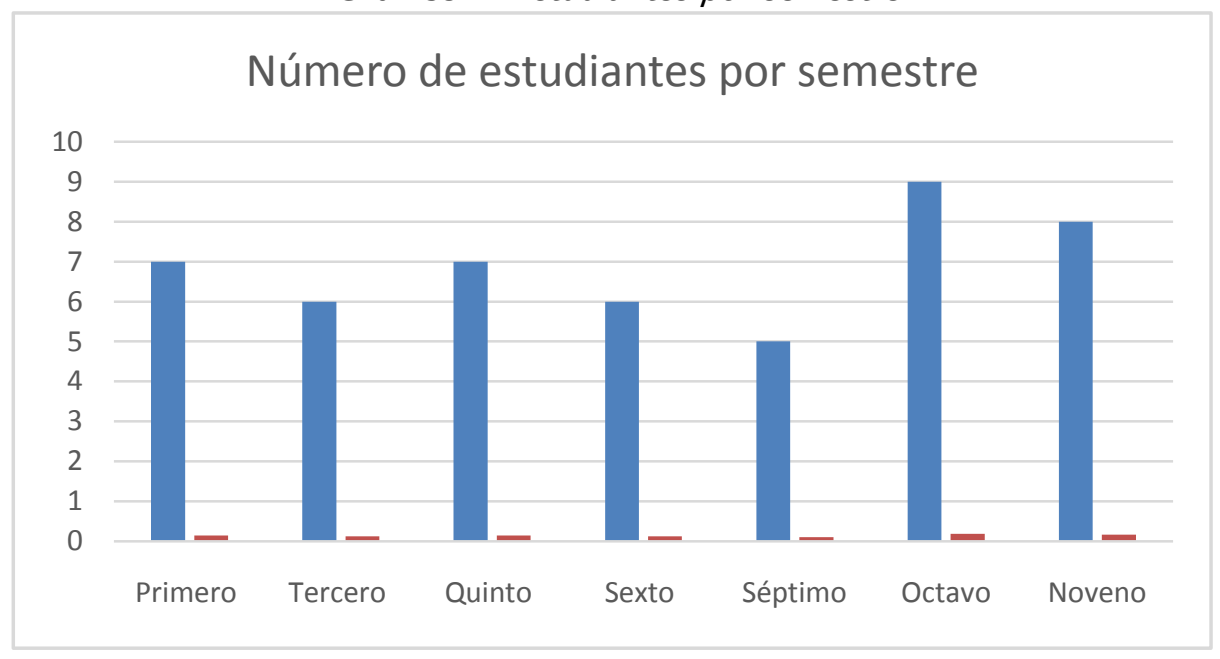

Fuente: Servicios Escolares UNACH (2018).

Con relación al género, 33.3\% del estudiantado son mujeres, en tanto que, $66.6 \%$ son varones, con una predominancia mayor de hombres formándose en esta profesión. El 
rango de edades oscila entre los 18 a 29 años, destacando con ello que hay una población que se integró tarde al nivel superior,

Respecto al origen de los estudiantes, $10.4 \%$ proviene de otros estados de la República mexicana y $89.5 \%$ tienen como lugar de origen el estado de Chiapas. Del total, $8.3 \%$ estudiaron el bachillerato en instituciones privadas y $91.6 \%$ en instituciones de carácter público. Este acercamiento resulta interesante puesto que puede comprenderse a partir del origen y contexto de su formación que proviene de hogares de clase media baja.

\section{Resultados}

\section{Tensiones y desigualdades en las interacciones áulicas}

Las interacciones se pueden observar a partir de las preguntas, declaraciones e interacciones silenciosas que encierran un código conocido exclusivamente por quienes lo han creado y lo comparten. El estudiantado, en general, mostraba comportamiento de poca disposición para colaborar en el salón de clases, justificándose en que no habían comprendido adecuadamente las indicaciones. Regularmente los profesores trabajan en torno a un esquema de repartir temas para que el estudiantado a lo largo del semestre los expongan y adicionalmente, en las clases consideradas como talleres, trabajan con algunas técnicas que los estudiantes deben conocer y dominar como la de clasificación y catalogación. Cuando algún profesor hace ver al estudiante la necesidad de colaborar más en clases, o qué debe leer más o que debe elegir su licenciatura de forma "correcta", los estudiantes se miran entre ellos y guardan silencio. Si el profesor los induce a la participación, insisten en que no comprenden sus lecturas o dan explicaciones confusas o superficiales al profesor.

Los estudiantes, en general, perciben una actitud de rechazo por parte de sus profesores, quienes los consideran como personas que han hecho malas elecciones vocacionales. Algunos miembros del profesorado suponen que estos estudiantes deben ser expulsados del programa por este espíritu pobre como bibliotecólogo.

Profesor 1: Ma1 ¿cómo te vendieron la idea de meterte a biblio?

Alumno1: Que el campo de la bibliotecología no estaba tan saturado, que había trabajo y por eso me metí.

Profesor 1: ¿No investigaste más? ¿Con qué se comía? ¿Solamente llegaste?

Alumno1: Ya estando adentro me gustó , pero solamente en el primer semestre tuve promedio alto y ahora llevo promedio de ocho. 
Observador: Manifiesta que se le hace difícil , ya lo siente aburrido; sin embargo, de manera contradictoria dice que ya le agrada. Entonces, la maestra escribe en el pizarrón "Bibliotecología Comparada" y de nuevo interroga al alumno si le gusta leer y él dice que muy poco; por lo que la maestra les dice que de todos los que ha entrevistado, casi nadie le gusta leer, que sólo leen por obligación. Mientras la Maestra interroga al alumno , algunos de sus compañeros ponen atención , pero otros escriben, otra está viend o su celular... Otra alumna explica que se metió a esta carrera porque le dijeron que era fácil, la Maestra ríe y le pregunta

Maestra: y ¿Cuál fue tu sorpresa?

Alumna: No he tenido sorpresas, todo ha sido fácil, pero en esta materia trataré de participar porque no es muy participativa, no me gusta leer, dice y... bueno tengo que leer... y... -contestó la alumna sin ningún cambio de actitud.

Asimismo, otro profesor señala que cuando tiene a cargo grupos de reciente ingreso, se reúne con sus colegas de semestre para discutir sobre la condición de los estudiantes que "valen la pena" y aquellos que deberían irse de la carrera. En un fragmento de su entrevista menciona:

Profesor 2: Bueno, mira -dice sacando su lista de asistencia y me enseña los nombres de cada uno de los alumnos y continua diciendo- yo creo que este grupo es bastante tranquilo, aunque hay alumnos que tienen muchos problemas, culturales, de lenguaje, por su condición muy humilde, pero a veces es necesario ser enérgico y a golpes y palabras se van componiendo. Luego comienza a hablar sobre el estudio que estoy haciendo, dice: Por eso estos trabajos como el que estás haciendo son muy interesantes y a mí me gustaría que lo dieras a conocer... - de nuevo retoma la plática - Creo que todos estos alumnos tienen una historia de vida, pero soy de la idea que si no dan el ancho que se vayan. Mira, Carlos, Adolfo y yo nos hemos sentado a analizar y por paquete hemos sacado a muchos que de plano no dan el ancho.

Por otra parte, con frecuencia, estos estudiantes señalan que los profesores no los motivan y por el contrario le hacen sentir que realizaron una mala elección, comentándoles que la Licenciatura no tiene campo de trabajo ni reconocimiento social y que se están formando para el desempleo. En las observaciones no hubo evidencia de este aspecto; sin embargo, los estudiantes afirman que los profesores si lo llevan a cabo; incluso señalan actitudes hacia ellos como si fueran personas poco capaces para cursar las asignaturas y con pocas posibilidades en su futuro laboral.

Con relación a este punto, existe un acercamiento entre profesores y estudiantes, por ser una población muy pequeña, lo cual permite que se conozcan entre si; propiciando una mejor convivencia entre estos actores educativos, al grado que es en la única licenciatura en donde se les da la bienvenida a los pocos estudiantes de nuevo ingreso. Se celebra el Día del Estudiante y otras celebraciones sociales en donde todos puedan convivir, incluso solicitando apoyo económico a los profesores. Existe, hasta cierto punto vista, desde algunos profesores una actitud paternalista, en la idea de que se sientan a gusto y en ningún momento piensen en desertar, puesto que estos estudiantes no llegan 
a esta licenciatura como primera opción, sino porque no tienen otra alternativa o porque no hay candados para ingresar.

A pesar de estos esfuerzos, existe inconformidad entre el estudiantado, que considera a la mayoría de sus profesores como arbitrarios, con clases aburridas y que faltan constantemente a clases. Sin embargo, esto solo queda a nivel de conversaciones no declaradas con las autoridades del plantel porque temen represalias en sus calificaciones.

Asimismo, dentro de los espacios áulicos, a partir de las observaciones realizadas, se pudo evidenciar que se sigue haciendo una práctica pedagógica tradicionalista casi en la totalidad de los profesores observados, se practican clases magistrales y exposiciones rutinarias en donde únicamente se privilegia el contenido, cuando bien les va a los estudiantes. Aunque la Universidad ofrezca cursos de formación docente, existe resistencia a formarse pedagógicamente para que su práctica docente sea más consciente.

A partir de las observaciones realizadas se evidenció que algunos profesores, en la idea de ser democráticos, ponen en las manos de los estudiantes la asignación de calificaciones, después de hacer una exposición tradicional. Esto representa un problema porque rara vez activan ideas previas y únicamente se dedican a repetir los contenidos, tal cual están plasmados y en ningún momento ponen o relacionan esta información con la realidad. Nunca durante las observaciones utilizaron ejemplos contextualizados para asociar los conocimientos teóricos con los empíricos. Simplemente se conforman con que los estudiantes repitan mecánicamente lo que dicen, las lecturas abordadas, adicionalmente a una pobre o casi inexistente lectura previa del estudiantado como se refiere en la siguiente observación:

Observador: Una de las estudiantes inicia diciendo.

Alumna 2: El tema que me tocó es la investigación histórica de la Bibliotecología.

Observador: Y comienza a exponer... Al minuto de su exposición el profesor la interrumpe explicando que hay un debate, entre sí se puede considerar una ciencia o un arte y luego le da la palabra de nuevo a la alumna. Esta lee lo que trae en sus diapositivas y cuando termina de leer la primera diapositiva, de nuevo el profesor le interrumpe y explica algo sobre el tema. Al terminar, de nuevo le da la palabra la alumna. Esta comienza a leer. De nuevo el profesor interrumpe diciendo que ella está hablando sobre las etapas de una investigación... El salón se encuentra a oscuras con las cortinas cerradas y las luces apagadas. Todos, alumnas y alumnos, permanecen sentados escuchando la intervención tanto de la compañera como del maestro, de manera intercalada. Aa- Po -Aa - Po... De pronto el profesor interrumpe la exposición y les comienza a platicar sobre las colecciones que tiene la biblioteca de la UNICACH, las cuales son antiquísimas, con materiales antiguos que pertenecen a nuestro estado. Les hace toda una semblanza platicándoles en qué consiste, cómo están y cómo se deben tratar porque ya están en situaciones graves por la misma antigüedad. De nuevo la alumna lee un párrafo y el maestro la vuelve a interrumpir... Después de hablar sobre lo que la alumna mencionó de nuevo le da la palabra. 
Profesor 3: Adelante, Bety - indica el profesor y la vuelve a interrumpir la forma de rescatar los documentos históricos es a través de la digitalización, indicando con esto que no significa que el libro vaya a desaparecer, pero muchas veces el contenido de una biblioteca puede estar en un disco almacenado sin la necesidad de tener metros y metros de espacio.

Observador: Tres alumnas que se encuentran atrás, hablan en voz baja, voltean a ver al observador y sonríen. Se ven distraídas, únicamente sentadas viendo a la ventana, se recarga una en el hombro de la otra... Mientras que la alumna sigue exponiendo, con la estrategia de leer textualmente lo que dicen las diapositivas. El profesor interrumpe nuevamente la exposición, pidiendo al grupo que responda tres preguntas que se relacionan con la historia de Chiapas: ¿Cuándo se dio el cambio de poderes? ¿Quién lo hizo? y ¿En dónde está?

Profesor 3: A ver tú que eres de San Cristóbal - le pregunta a una alumna en particular.

Alumna 4: No lo sé profesor - le responde.

Profesor 3: ¿Lo digo? iReflexionen! iPiensen! - Menciona sin encontrar respuesta alguna, por lo que retoma nuevamente la palabra. Fue a finales del Siglo XIX, en 1892, siendo Gobernador Emilio Rabasa, se encuentra en el Archivo Histórico del Estado, pero lo interesante es que ha sido rescatado y ahora se encuentra legible ya que lo han digitalizado - y deja de hablar, permitiendo de nuevo a la alumna que intervenga para concluir su tarea que consiste en leer las diapositivas.

Alumna 3: Es todo profe - dice cuando concluye.

Profesor 3: Bien ¿Alguna pregunta? ¿Nadie?

Alumna 5: Profe quiero saber si para hacer investigación tengo que seguir todas las etapas.

Observador: La alumna que expuso contesta

Alumna 3: iSí! Porque no puedes hacer un pastel sin poner los ingredientes antes.

Profesor 3: iExacto! Bueno, si no hay preguntas saquen su hoja para hacer la evaluación correspondiente a la expositora. Se van a evaluar los siguientes puntos: material utilizado, dominio del tema y lenguaje utilizado.

Observador: Todos los estudiantes sacan su hoja y escriben para evaluar la exposición de la alumna. Mientras, la alumna comienza a apagar el equipo.

Profesor 3: iListos! ¿Quién empieza? - todos permanecen callados, hasta que una de las estudiantes pide la palabra- iAdelante!

Alumna 6: En el material utilizado le pongo nueve, porque utilizó la tecnología. En dominio del tema ocho, porque se vio que no domina el tema, leyó mucho y el lenguaje utilizado fue bueno, le pongo nueve.

Observador: Así, sucesivamente todos los estudiantes evaluaron a la alumna 
dándole por mayoría nueve y diez en tecnología, en dominio ocho y entre ocho y nueve en lenguaje utilizado.

Como se pudo leer, realmente no se profundiza en el análisis del conocimiento, aunado a que el profesor interrumpe constantemente las exposiciones de los estudiantes y quizás únicamente se da como una mera receta para que conozcan los pasos, en este caso de cómo hacer una investigación por ensayo error. Sobre todo, si se toma en cuenta que hacer evaluaciones entre pares y a partir de la subjetividad de los estudiantes, propicia desigualdades.

\section{Enunciados-contenido e intención del mensaje}

Los estudiantes consideran que los profesores tienen una mala imagen de ellos, al señalarles que han hecho malas elecciones vocacionales y lo confirman cuando algunos docentes suponen que estos estudiantes deben ser expulsados del programa por este espíritu pobre como bibliotecólogos. Esto se expresa en comentarios de profesores en los pasillos de la Facultad y pláticas informales donde indican que al inicio de cada semestre hacen una especie de predicción acerca de los alumnos que deben permanecer en el programa y los que deben irse, a partir de la interacción que han tenido con ellos en las primeras sesiones en el salón de clases. Esto particularmente lo realizan con los que ingresan a primer semestre de la Licenciatura.

La consecuencia de esta apreciación por parte de los profesores se puede identificar cuando los estudiantes manifiestan que los profesores no los motivan y, por el contrario, le hacen sentir que hicieron una mala elección porque es una Licenciatura que no tiene campo de trabajo ni reconocimiento social, y que deberían tomar en cuenta que como estudiantes se están formando para el desempleo. Sin embargo, esta Licenciatura es la única que se oferta en el Sur-Sureste de la República mexicana. De acuerdo con lo que señala el Plan de Estudios de esta Licenciatura (UNACH, 1992).

En las observaciones no hubo evidencia de este aspecto, sin embargo, los estudiantes afirman que los profesores si lo llevan a cabo, incluso señalan que los hacen sentir que es difícil cursar las asignaturas y que tienen pocas posibilidades en su futuro laboral.

\section{La mirada de los profesores sobre sus estudiantes}

La selectividad de los profesores se basa en el éxito académico de sus estudiantes y la imagen del modelo a seguir. En ese sentido Rockwell (1995) hace alusión que los profesores, cuando se reúnen en grupos informales y en conversaciones menos públicas, llevan a cabo interacciones entre pares, con la idea de contarse anécdotas de experiencias que se les han presentado durante el desarrollo de su labor docente en cada uno de los grupos que les toca trabajar. Esto da paso a la construcción de concepciones alternativas, las cuales de manera institucional, se conciben generando la reproducción de una gran diversidad de tradiciones. Fuera del aula, las conversaciones de los profesores sobre sus estudiantes se parecen a las del siguiente extracto: 
Observador: ¿Oye ese trabajo te lo entregan? -refiriéndose al trabajo que hicieron en el salón.

Profesor 3: Sí, claro. Es que me interesa sobre todo el trabajo de Luis Francisco, porque se me hace un niño muy dedicado.

Observador: Pues sí.

Profesor3: Mira, Jacobo, al principio quiso que por ser indígena le tuvieran lástima, hasta que yo le dije, momento, si yo también soy "indígena". Mi papá fue campesino y pobre y eso no impidió que me superara.

Observador: Claro- le contesto y concluyo la conversación porque llega otra profesora a interrumpirnos, por lo que el maestro se despidió.

Este extracto de observación conlleva a reflexionar que se presentan diversas formas de interacción entre profesores y estudiantes, las cuales pueden variar a partir de ciertas características de personalidad en cada uno de ellos, los cuales pueden mostrarse formales, agresivos, afectuosos, tolerantes o desiguales, todo esto dependiendo del contexto en que se desarrolle tal interacción. En el caso particular de la observación antes citada, se puede observar a un profesor parcialmente tolerante.

\section{Los estudiantes participando en clases}

Las clases se convierten en rutina para el resto de los profesores, quienes desde el primer día, como tradición se dedican a organizar equipos y repartir el contenido en temas para que los estudiantes se hagan cargo de las exposiciones. Estas consisten en copiar y pegar textualmente en las diapositivas el contenido de los textos y leerlas con una breve explicación, como se pudo observar, pocos profesores retroalimentaban las exposiciones. O incluso la exposición del profesor de algún contenido en donde se considera experto sin que deba ser cuestionado, por ejemplo:

Alumno1: ¿Profe, por qué en un número dice del corazón y en otro número dice Corazón? - dice refiriéndose a la clasificación temática.

Maestra 2: Busca en el libro... ¿Ya desglosaste la clasificación? Pasen a escribir la clasificación que tienen por equipo. A ver Julia.

Alumna 2: No soy Julia, soy Arely.

Maestra 2: A ver allá, Carlos, Omar, Citlali.

Observador: Los alumnos contestan un número y la profesora continúa revisando.

Con respecto a los conocimientos previos de los estudiantes para distinguir entre el código restringido y elaborado, se pudo observar en la siguiente interacción, donde el 
profesor con el poder legítimo que le fue conferido por la escuela, hace sentir a los estudiantes que no poseen los conocimientos suficientes para hacerse cargo del contenido de la asignatura que imparte:

Maestra 3: ¿Karla, hiciste la tarea?

Alumna1: Más o menos, es que no le entendía.

Maestra 3: Yo no les dejo tarea para que se somaten analizándola, porque esto es un asunto de encabezamiento de materia que ya deben saber, dice mientras que un alumno escribe en el pizarrón la sigla de IPN.

Alumno 1: ¿Instituto Politécnico Nacional?

Maestra 3: Si.

Alumno 2: Ya le habíamos dicho.

Maestro 3: Si pero hablan muy bajito.

Alumno 3: Profe, tengo una duda ¿Esto lo íbamos a hacer únicamente por lógica?

Maestra 3: Es que para hacerlo tienen que tener mucho conocimiento amplio.

Alumno 4: Es que no sabemos cómo hacerlo entonces.

Maestra 3: Ah sí, tienes razón tienes que analizarlo.

Con todo esto, se puede apreciar que algunos de los profesores, denotan falta de formación disciplinaria y que al momento de ser cuestionado por los estudiantes, tratan de dar argumentos poco convincentes, dando margen a que los estudiantes comenten tanto cuestiones positivas como negativas del desempeño de los profesores como de sus propios compañeros, porque, entre los estudiantes existe un margen mucho más amplio de interacciones denominadas horizontales y en los pasillos, de manera informal, comentan de las aptitudes, actitudes y formas de comportamiento de sus profesores.

\section{Discusión}

El interior de las aulas se parece a una caja negra de los aviones, en la cual se conoce que funciona, pero pocos conocen lo que sucede adentro. Las aulas son espacios privilegiados de formación y rico en interacciones entre estudiantes y profesores para comprender el proceso enseñanza aprendizaje en la educación superior. Sin embargo, la mirada investigativa permite analizar las interacciones profesor-alumno con relación al conocimiento en el que se está formando.

Los conocimientos previos de estudiantes y profesores se activan en las interacciones áulicas y deberían servir de referente para los nuevos aprendizajes, sin embargo, una 
serie de condiciones revelan accesos al conocimiento en situación de desigualdad, lo que provoca una posibilidad casi nula de comunicación del estudiantado, frente a un clima de imposición de una cultura escolar que en parte les es ajena porque el código es elaborado (Bernstein \& Cook-Gumperz, 1973).

Las tensiones que se generan ante la posibilidad de comunicarse para comprender el conocimiento disciplinario trae consecuencias expresadas de diversas formas, por ejemplo, en una especie de apatía o insatisfacción por parte del estudiantado quienes van construyendo una mirada negativa sobre sí mismos, que es legitimada por la autoridad pedagógica del profesor (Bourdieu, 1996). El habitus incorporado resulta insuficiente desde el punto de vista de los profesores, quienes desde el imaginario de alumnos ideales que en la realidad no existen. Es evidente que existe una brecha casi insalvable entre el profesorado y el estudiantado en términos de la insatisfacción que se manifiesta de forma latente en la interacción alumno-alumno.

Aunque poco se ha desarrollado esta idea a lo largo de la investigación, no se puede soslayar el asunto de la sociedad mexicana fuertemente mercantilizada y en proceso de credencialización educativa (Blanco, 2017), ya que esto permite comprender la estancia en las aulas de estudiantes que eligen la formación de Bibliotecología como una forma de acceder en la movilidad social vía la educación superior, más allá del deseo de ser un profesional calificado en esta área.

Por su parte, el profesorado, se considera limitado en cuanto a las estrategias de enseñanza para un aprendizaje significativo, debido a la pobre mirada de sus estudiantes con una práctica pedagógica monótona y con poco espacio para andamiar la formación de estos últimos; por lo que en las aulas se reproducen las condiciones de desigualdad, donde puede decirse que hay estudiantes de primera y de segunda, de acuerdo a la visión que tenga el profesorado.

\section{Conclusión}

La desigualdad, como se ha revisado a lo largo del artículo, es un problema complejo que tiene que ver desde dos grandes aristas: una desde un nivel macro y la otra desde un nivel micro, puesto que intervienen muchos factores tanto externos como internos. Es en la conjunción de estos niveles donde se propician y reproducen las desigualdades sociales que, como ya se ha revisado, no depende exclusivamente del entorno inmediato social sino que abarca aspectos de carácter social.

Todo esto comunica a la sociedad parámetros de clasificación, desde la jerarquía de calificaciones propuesta por Perrenoud (2008), propiciando incluso que entre instituciones sociales se sigan marcando desigualdades sociales, a partir de insistir en tratar por igual a los desiguales. Sin embargo, este fenómeno de reproducción se observa en el interior de las aulas, con estudiantes que poseen códigos lingüísticos restringidos, reforzados a lo largo de su formación que no es suficiente para apuntalar los nuevos conocimientos. 
Se agrava aún más con las exigencias de una educación de calidad, en donde se sustituyen los valores de justicia, equidad, dignidad humana, solidaridad y distribución de la riqueza y del capital cultural por la preocupación de la eficacia, competitividad, además de la excelencia, con una mirada de la educación como consumo (Gimeno, 2001), que puede dar lugar a problemas inherentes a una formación profesional poco sólida.

La elección de una profesión, implica pensarla desde los imaginarios sociales que se construyen y que inciden en la forma en que el estudiantado la perciba como algo donde tendrá éxito. En el caso de las Ciencias Sociales y Humanidades, todavía existen algunas concepciones erróneas, concibiéndolas como más fáciles que las llamadas Ciencias Exactas. El problema es que desde allí, se asume como buen o mal estudiante o buen o mal profesionista. La Bibliotecología no es la excepción, cuando se compara con otras ciencias en el ámbito de lo cotidiano.

Por otra parte, los resultados muestran evidencias con respecto a la urgencia de procesos de formación docente que puedan reflexionar sobre lo educativo y puedan comprender la importancia que tiene el desarrollo de estrategias didácticas para favorecer el interés, la motivación para coadyuvar a mejorar las condiciones de aula con respecto al trato entre iguales.

Es importante que el profesorado pueda identificar sus fortalezas, pero también sus áreas de oportunidad en torno a la práctica docente que desarrolla en las aulas. Debe destacarse su papel con estudiantes que requieren fuerte apoyo para introducirlos paulatinamente al código académico elaborado, sin menoscabar su origen social.

\section{Referencias}

Austin, J. L. (1981). Cómo hacer cosas con palabras. Barcelona: Paidós.

Bartolucci, J. (1994). Desigualdad social, educación superior y sociología en México. México: Porrúa.

Bernstein, B. y Díaz, M. (1985). Hacia una teoría del discurso pedagógico. Revista Colombiana de Educación, 8(17), 1-18. Recuperado de http://revistas.pedagogica.edu.co/index.php/RCE/article/view/5120/4199

Bernstein, B. \& Cook-Gumperz, J. (1973). The coding grid, theory and operations. En: Cook-Gumperz, J., (comp.). Social control and socialization: a study of social class differences in the language of maternal control (pp. 48-72). Londres: Routledge y Kegan Paul.

Blanco, E. (2017) Teoría de la reproducción y desigualdad educativa. Evidencia para el nivel primario. Revista Mexicana de Investigación Educativa, 22(74), 751-781. Recuperado de http://www.scielo.org.mx/pdf/rmie/v22n74/1405-6666-rmie-22-7400751.pdf 
Bourdieu, P. y Passeron, J. (1996). La reproducción. Elementos para una teoría del sistema de enseñanza. Barcelona: Fontamara.

Buendía, A., Acosta, A. y Gil Antón, M. (2019). En busca de un rostro. (In) visibles pero siempre presentes. Revista Mexicana de Investigación Educativa, 24(80), 15-41. Recuperado de http://www.scielo.org.mx/pdf/rmie/v24n80/1405-6666-rmie-24-8015.pdf

Capocasale, A. (2015). La investigación cualitativa. Aproximación a algunos de sus métodos de investigación. En: Abero, L., Berardi, L., Capocasale, A., Montejo, S. y Rojas, R. Investigación Educativa. Abriendo Puertas al conocimiento. Uruguay: Camus Ediciones. Recuperado de http://biblioteca.clacso.edu.ar/clacso/se/20150610045455/InvestigacionEducativa.pdf

Carrillo, J. (1982). La sociología, Teorías, Métodos, Tecnicismos y problemas sociales México: Jocamar.

Chacón, R. (2003). Elección de carrera y formación Profesional en el caso de la licenciatura en bibliotecología de la UNACH. Tesis de maestría en Educación Superior Universidad Autónoma de Chiapas, México.

Feito, R. (s/f) Teorías sociológicas de la educación. Universidad Complutense Madrid. Recuperado de http://webs.ucm.es/BUCM/cps/lecturas/4.htm

Gimeno-Sacristán, J. (2001). Políticas y prácticas culturales en las aulas: los abismos de la etapa postmoderna. Revista Fundamentos en Humanidades, I (4), 7-43. Recuperado de http://www.redalyc.org/articulo. oa?idp=1\&id=18400401\&cid =460

Gil-Antón, M. (6 de Junio de 2016). Sobre el fracaso de la reforma educativa: entrevista a Manuel Gil Antón. Horizontal, pág. 7. Recuperado de https://horizontal.mx/sobre-elfracaso-de-la-reforma-educativa-entrevista-a-manuel-gil-anton/

Goetz, J. y Lecompte, M. (1988) Etnografía y diseño cualitativo en investigación educativa. Madrid: Morata.

Lera, J., Martínez, J., Ochoa, R. y Rivas, J. (2017). Desigualdad social y educativa en México y España. Nuevas formas de atender el desarrollo. Revista Internacional de Ciencias Sociales y Humanidades, XXVII (1), 133-161. Recuperado de https://www.redalyc.org/pdf/654/65456040009.pdf

Lutte, G. (1991). Liberar la Adolescencia. La Psicología de los Jóvenes de Hoy. Barcelona: Herder.

Malinowski, B. (1995) Los argonautas del Pacífico Occidental. Barcelona: Península.

Márquez, A. (2016) La desigualdad social y las reformas educativas. Revista Perfiles, Educativos, XXXVIII (154). 3-18. Recuperado de http://www.scielo.org.mx/scielo.php?script=sci_arttext\&pid=S018526982016000400001 
Martínez-Rizo, F. (2012). Contextos vulnerables: las aportaciones de la evaluación. Revista Bordon (2) 41-50. Recuperado de https://recyt. fecyt.es/index.php/BORDON/article/view/21986

Maturana, G. y Garzón, C. (2015) La etnografía en el ámbito educativo: una alternativa metodológica en la investigación al servicio docente. Revista Educación y Desarrollo Social, 9(2), 193-205. Recuperado de https://dialnet.unirioja.es/servlet/articulo?codigo=5386223

Mauss, M. (1974) Introducción a la etnografía. Madrid: Itsmo.

Parsons, T. (1966). Estructura y proceso en las sociedades moderna. Madrid: Instituto de Estudios Políticos.

Perrenout, Ph. (2008). La construcción del éxito y fracaso escolar. Madrid: Morata.

Perret-Clermont, A. N. (1979). Procesos psicosociológicos y fracaso escolar. Infancia y Aprendizaje, (6), 3-13. Recuperado de https://www.researchgate.net/publication/49251545_Procesos_Psicosociologicos_y_Fra caso_Escolar

Redondo, J. (1997). La dinámica escolar: de la diferencia a la desigualdad. Revista de Psicología, (6), 7-18. Doi: http://dx.doi.org/10.5354/0719-0581.2012.18656

Rockwell, E. (1995). Dimensiones de la experiencia escolar. México: Fondo de Cultura Económica.

UNACH (1992). Plan de Estudios de la Licenciatura en Bibliotecología. Facultad de Humanidades. Chiapas, México: Universidad Autónoma de Chiapas.

UNACH (2018). Anuario Estadístico 2018. Chiapas, México: Universidad Autónoma de Chiapas.

UNESCO (2011). La UNESCO y la educación. Toda persona tiene derecho a la educación. París:

UNESCO.

Recuperado

de https://unesdoc.unesco.org/ark:/48223/pf0000212715_spa 